Article

\title{
Modeling and Analysis of Resonance in LCL-Type Grid-Connected Inverters under Different Control Schemes
}

\author{
Yanxue $\mathrm{Yu}^{1}$, Haoyu $\mathrm{Li}^{1, *}$, Zhenwei $\mathrm{Li}^{2}$ and Zhou Zhao ${ }^{1}$ \\ 1 School of Electrical Engineering and Automation, Harbin Institute of Technology, Harbin 150001, China; \\ yuyanxueppkz@163.com (Y.Y.); halbertboy@gmail.com (Z.Z.) \\ 2 College of Automation \& Electronic Engineering, Qingdao University of Science \& Technology; \\ Qingdao 266000, China; lizw@qust.edu.cn \\ * Correspondence: lihy@hit.edu.cn; Tel.: +86-451-8641-2811 \\ Academic Editor: Gabriele Grandi \\ Received: 1 November 2016; Accepted: 10 January 2017; Published: 16 January 2017
}

\begin{abstract}
As a basic building block in power systems, the three-phase voltage-source inverter (VSI) connects the distributed energy to the grid. For the inductor-capacitor-inductor (LCL)-filter three-phase VSI, according to different current sampling position and different reference frame, there mainly exist four control schemes. Different control schemes present different impedance characteristics in their corresponding determined frequency range. To analyze the existing resonance phenomena due to the variation of grid impedances, the sequence impedance models of LCL-type grid-connected three-phase inverters under different control schemes are presented using the harmonic linearization method. The impedance-based stability analysis approach is then applied to compare the relative stability issues due to the impedance differences at some frequencies and to choose the best control scheme and the better controller parameters regulating method for the LCL-type three-phase VSI. The simulation and experiments both validate the resonance analysis results.
\end{abstract}

Keywords: different control schemes; impedance; LCL-type grid-connected inverters; resonance analysis

\section{Introduction}

As important interfaces between distributed power generation systems and a power grid, three-phase voltage-source inverters are widely used in grid integration of renewable energy [1]. Due to the increasingly complexity of the dynamics of the power grid [2] and the varying differences among inverters [3], various stability issues exist in the inverter-grid system. The interaction between the inverter output impedance and the grid impedance is a particular issue that can trigger resonance and control instability of grid-connected inverters [4]. Ideally, the output impedance of the inverter is infinite and the impedance of the grid is zero, so there is no coupling between them [5]. However, to the inverter, its output impedance can be confined by the filter and control loop; to the grid, due to the long distance transmission cables, it may feature as the weak grid containing a large set of impedances, which could trigger resonances in the inverter-grid systems [6]. Therefore the grid impedance affects the robust stability of grid-connected inverters and it's essential to analyze the resonant problems and improve the stability margin from the point of the output impedances of VSIs [1,2,5,7-10].

Considering the VSI, its output impedance characteristics would be different in terms of different output filters. Compared to the L-type filter, the LCL-type filter has been widely used in grid-connected inverters due to its higher attenuating ability [11]. However, it needs additional damping methods to eliminate the high-frequency resonances. Apart from that, current control is another important issue for the LCL-filter VSIs [12]. Unlike L-filter VSIs whose controlled current is sampled from the inductor; 
to LCL-filter VSIs, both the inverter-side current (IC) and grid-side current (GC) can be sampled and controlled, which means that the stability issues of the LCL-type grid-connected inverters would be more complicated under different sampling positions. Although the stability issues of the LCL-type grid-connected inverters have been widely studied in the literature [12-17], in the weak grid, most of the studies focus on relevant damping methods to eliminate the resonance resulting from large grid impedance, mainly including the multi-loop control method [13], the filter-based active damping method [14,15] and the virtual impedance method [16]. Among the various active damping solutions, capacitor-current-feedback damping has been widely used for its ease of implementation [12,13]. When it comes to the stability comparison on different current sampling position of LCL-filter, the studies are quite limited. In [12], based on a general mathematical model, a comparative analysis of different control schemes (namely, the grid current control, the inverter-side inductor current control, and the weighted average current control) was carried out in terms of the grid current stability. It revealed that when the inverter-side inductor current is controlled, the grid current shows the same stability as the inverter-side inductor current; but when the weighted average current is controlled, both the grid current and the inverter-side inductor current are critically stable even though the weighted average current can be easily stabilized. Additionally, in [14], the relationship between current sensor positions and LCL-filter resonance frequencies without active damping is analyzed by means of open-loop Bode diagram. It's concluded that for the GC sampling, the system is stable for the higher resonance frequencies and for the IC sampling, the system is stable for the lower resonance frequencies. In conclusion, there is no detailed model analysis aiming at comparing different control schemes of LCL-filter VSIs.

In a three-phase VSI, the current can be controlled in a rotating $d q$-domain reference frame or a stationary $\alpha \beta$-domain reference frame, most of which use a proportional-integral (PI) controller and proportional-resonant (PR) controller, respectively. Different reference frames show some differences in terms of the corresponding output impedances [1], so it is necessary to compare their robustness under weak grid conditions. However, the existing literature paying attention to this problem is too limited. Reference [1] emphasizes on the modeling of output impedances of L-type VSI in $d q$-domain and phase-domain reference frame, and there is no relevant comparison for a weak grid. In [18], three different current controllers' performance are compared just by time-domain simulation, including the PI-controller in synchronous rotating reference frame, the PR-controller in the stationary reference frame and the phase current hysteresis controller. The results indicated that the PR-controller is more adaptable to the grid impedance variation. Similarly, in [19], the same kind of controllers as in [18] were compared on the basis of the steady state error produced, transient performance, harmonic content and hardware implementation aspects in virtual synchronous machines. Additionally, in [20], the performance of a PR controller is compared with that of the PI controller just by time-domain simulation and the authors draw the conclusion that the PR controller achieves better reduction in total harmonic distortion (THD) in the current signal spectrum.

Despite the extensive literature that has been published on the stability analysis of LCL-filter three-phase VSIs under weak grid conditions, detailed mathematical models and differences among different control schemes, including different current sampling position and different reference frame remain unrevealed. Research on stability improvement approaches under the control scheme with better robustness will be more efficient to solve the resonance issues, therefore it's quite necessary to ensure a control scheme with greater robustness for the weak grid situation.

In order to choose the best control scheme for the LCL-filter VSI under weak grid conditions among the most common ones, including the $\alpha \beta$-domain GC feedback control, $\alpha \beta$-domain IC feedback control, $d q$-domain GC feedback control, and $d q$-domain IC feedback control, this paper models their sequence impedances using the harmonic linearization method and analyzes their functionary mechanism from the point of impedances to explain a variety of existing resonance phenomena. 


\section{Impedance Modeling and Analysis}

The grid-connected VSI with LCL-filter is depicted in Figure 1. Phase voltages are denoted as $v_{\mathrm{a}}, v_{\mathrm{b}}, v_{\mathrm{c}}$, while phase currents are represented by $i_{\mathrm{a}}, i_{\mathrm{b}}, i_{\mathrm{c}} . L_{1}$ and $L_{2}$ are the inverter-side inductor and grid-side inductor, respectively. $C_{f}$ is the filter capacitor. The capacitor current feedback loop is chosen to eliminate the high-frequency resonance resulting from the LCL filter, $K_{i}$ is the feedback coefficient. $Z_{0}$ denotes the inverter impedance and $Z_{g}$ denotes the grid impedance. $L_{g}$ denotes the inductive component of the grid. Figure 1 shows the $d q$-domain GC feedback control. With regard to other control schemes, Figure 2 depicts the IC feedback control, here $C_{f}$ and $L_{2}$ are considered as parts of the grid impedance expressed as $Z_{g}$. Figure 3 shows the $\alpha \beta$-domain current feedback control.

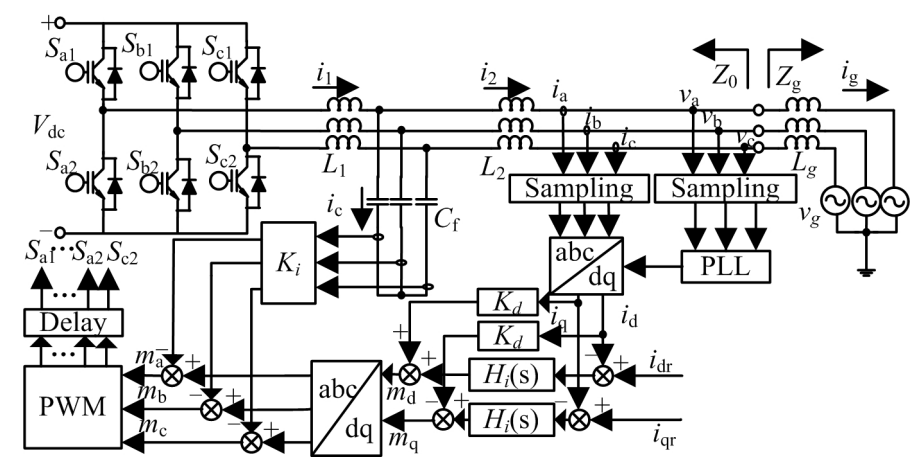

Figure 1. Block diagram of LCL-type inverter with $d q$-domain GC feedback for grid-connected applications.

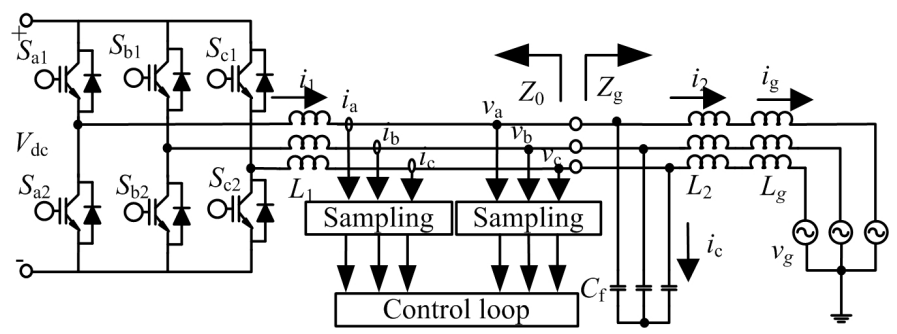

Figure 2. Block diagram of LCL-type inverter with IC feedback.

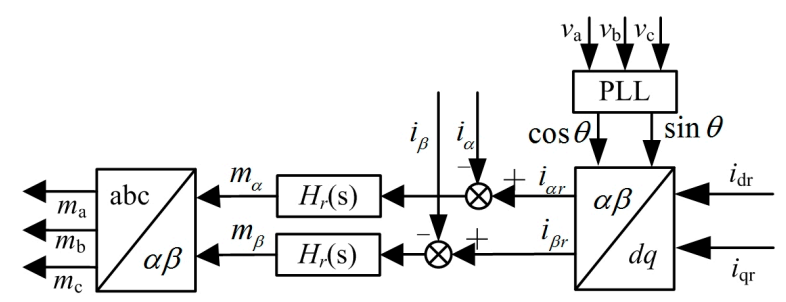

Figure 3. Block diagram of a current controller with $\alpha \beta$-domain current feedback.

The harmonic linearization impedance modeling approach is based on the Fourier Transform. Specifically, apply a small positive- or negative-sequence voltage perturbation on the fundamental voltage at the point of common coupling (PCC) and find the corresponding response of the inverter currents at the same frequency of the superimposed perturbation. Then the impedance is defined as the ratio of the perturbed voltages to the corresponding currents [10]. Here we take the modeling of positive-sequence impedance under $d q$-domain GC feedback control as an example to introduce the modeling approach. 


\subsection{Impedance Modeling}

According to the harmonic linearization impedance modeling approach and the block diagram depicted in Figure 1, when applying a small positive-sequence voltage perturbation on the fundamental voltage, we can get the positive-sequence signal flow diagram of inverter with $d q$-domain GC feedback control shown in Figure 4. In this figure $u_{k}(\mathrm{~s})$ is the output voltage at the inverter ac terminal, $u_{c k}(\mathrm{~s})$ is the output voltage at the capacitor ac terminal, $u_{g k}(\mathrm{~s})$ is the output voltage at the grid ac terminal. $m$ is the modulating signals for PWM, $K_{m}$ is the modulator gain. $G_{v}(\mathrm{~s})$ and $G_{i}(\mathrm{~s})$ model the voltage and current sampling delay. $V_{d c}$ is the dc bus voltage, $I_{d r}$ and $I_{q r}$ are the reference currents. In modeling, $V_{d c}, I_{d r}, I_{q r}$ is assumed constant. $f_{1}$ and $f_{p}$ represent the frequencies of the signal and $f_{p}$ is the frequency of positive-sequence perturbation.

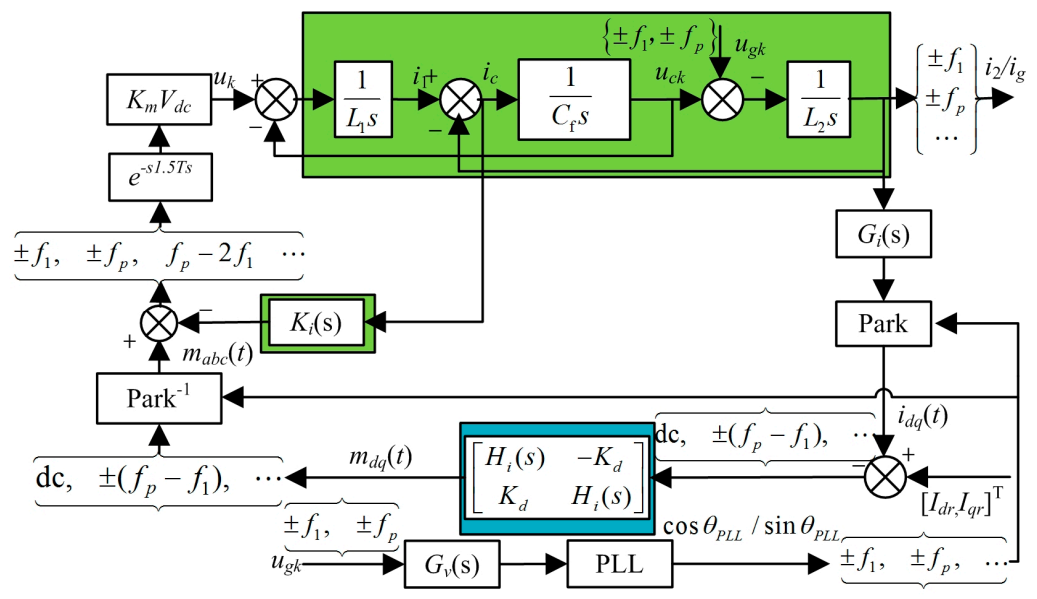

Figure 4. Signal flow diagram of inverter with $d q$-domain GC feedback control during positivesequence perturbation.

In the time domain, the phase voltages with a small-signal positive perturbation can be written as:

$$
\left.v_{a}(t)=V_{1} \cos \left(2 \pi f_{1} t\right)+V_{p} \cos \left(2 \pi f_{p} t+\right] \phi_{v p}\right)
$$

where $V_{1}$ with $f_{1}$ is the amplitude and frequency of the fundamental voltage; $V_{p}, f_{p}$ and $\phi_{v p}$ is the amplitude, frequency and phase of the positive-sequence voltage perturbation. In the frequency domain, (1) can be written as:

$$
V_{a n}[f]=\left\{\begin{array}{l}
V_{1 n}[f], f= \pm f_{1} \\
V_{p n}[f], f= \pm f_{p}
\end{array}\right.
$$

where $V_{a n}[f]$ is the Fourier transform of $v_{a}(\mathfrak{t}), V_{1 n}[f]$ and $V_{p n}[f]$ are defined as:

$$
V_{1 n}[f]=\frac{V_{1}}{2}, V_{p n}[f]=\frac{V_{p}}{2} e^{ \pm j \phi_{v p}}
$$

Other phase voltages can be inferred from (2):

$$
V_{b n}[f]=\left\{\begin{array}{l}
V_{1 n}[f] e^{\mp j \frac{2}{3} \pi}, f= \pm f_{1} \\
V_{p n}[f] e^{\mp j \frac{2}{3} \pi}, f= \pm f_{p}
\end{array} V_{c n}[f]=\left\{\begin{array}{c}
V_{1 n}[f] e^{ \pm j \frac{2}{3} \pi}, f= \pm f_{1} \\
V_{p n}[f] e^{ \pm j \frac{2}{3} \pi}, f= \pm f_{p}
\end{array}\right.\right.
$$

In order to find the current response under the positive-sequence voltage perturbation shown in (1), the first step is to derive the small-signal response of the phase-locked loop (PLL).

Figure 5 depicts the basic building block of a synchronous reference frame (SRF) PLL, where $\theta_{P L L}$ is the phase of the grid voltage, and $H_{P L L}(\mathrm{~s})$ is the loop compensator. In order to solve the 
nonlinearity in Park's transformation, we break Figure 5 into two parts as depicted in Figure 6, where $\Delta \theta=\theta_{P L L}(t)-\theta_{1}(t)$.

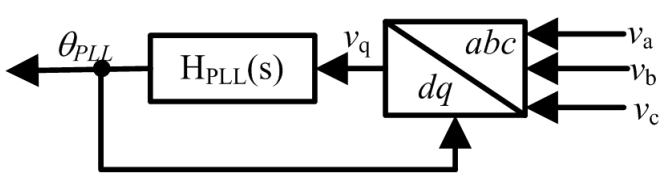

Figure 5. Block diagram of a SRF-PLL.

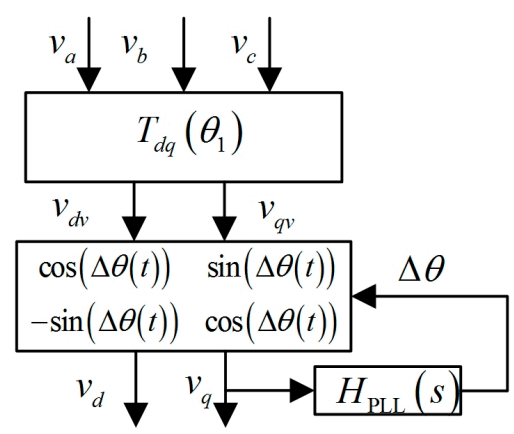

Figure 6. Block diagram of a decomposed SRF-PLL.

Introduce the frequency-domain Equations (2) and (4) into Figure 6, the calculated lock signal in the frequency domain is written as follows:

$$
\begin{gathered}
\cos \theta_{P L L}[f]=\frac{1}{2} \frac{H_{P L L}\left(s-j 2 \pi f_{1}\right) G_{v}(s)}{1+V_{1} H_{P L L}\left(s-j 2 \pi f_{1}\right)} V_{p n}(s) f= \pm f_{p} \\
\sin \theta_{P L L}[f]=\mp j \cos \theta_{P L L}[f] f= \pm f_{p}
\end{gathered}
$$

Finally the following transfer function is found:

$$
T_{p}(s)=\frac{1}{2} \frac{H_{P L L}\left(s-j 2 \pi f_{1}\right) G_{v}(s)}{1+V_{1} H_{P L L}\left(s-j 2 \pi f_{1}\right)} f= \pm f_{p}
$$

where $T_{p}(\mathrm{~s})$ models the response of $\cos \left(\theta_{P L L}(t)\right)$ to $V_{p}(\mathrm{~s})$.

Similarly, for a negative-sequence perturbation, the transfer function is modeled as:

$$
T_{n}(s)=\frac{1}{2} \frac{H_{P L L}\left(s+j 2 \pi f_{1}\right) G_{v}(s)}{1+V_{1} H_{P L L}\left(s+j 2 \pi f_{1}\right)} f= \pm f_{n}
$$

Introducing the small-signal response of PLL into Figure 4, we can get the inverter output impedances under $d q$-domain GC feedback control as in (9) and (10):

$$
\begin{gathered}
Z_{p}{ }^{G C}(s)=\frac{K_{m} V_{d c} e^{-s 1.5 T_{s}}\left[H_{i}\left(s-j 2 \pi f_{1}\right)-j K_{d}\right] G_{i}(s)+P_{1} G C(s)}{P_{2}{ }^{G C}(s)-K_{m} V_{d c} e^{-s 1.5 T_{s}}\left[H_{i}\left(s-j 2 \pi f_{1}\right)-j K_{d}+M_{n G C}[d c] / I_{1}\right] T_{p}(s) I_{1}} \\
Z_{n}{ }^{G C}(s)=\frac{K_{m} V_{d c} e^{-s 1.5 T_{s}}\left[H_{i}\left(s+j 2 \pi f_{1}\right)+j K_{d}\right] G_{i}(s)+P_{1} G C(s)}{P_{2} G C(s)-K_{m} V_{d c} e^{-s 1.5 T_{s}}\left[H_{i}\left(s+j 2 \pi f_{1}\right)+j K_{d}+M^{*}{ }_{n G C}[d c] / I_{1}{ }^{*}\right] T_{n}(s) I_{1}{ }^{*}}
\end{gathered}
$$

where:

$$
\begin{gathered}
P_{1}^{G C}(s)=L_{1} L_{2} s^{2}\left(s C_{f}+\frac{1}{R_{p d}} e^{-s 1.5 T_{s}}\right)+\left(L_{1}+L_{2}\right) s \\
P_{2} G C(s)=L_{1} s\left(C_{f} s+\frac{1}{R_{p d}} e^{-s 1.5 T_{s}}\right)+1
\end{gathered}
$$




$$
\begin{gathered}
R_{p d}=\frac{L_{1}}{K_{i} K_{m} C_{f}} \\
M_{n G C}[d c]=\frac{V_{1} P_{2}{ }^{G C}\left(j 2 \pi f_{p}\right)+I_{1} e^{j \phi_{i 1} P_{1} G C}\left(j 2 \pi f_{p}\right)}{K_{m} V_{d c} e^{-s 1.5 T_{s}}}
\end{gathered}
$$

and $M_{n G C}{ }^{*}$ is the complex conjugate of $M_{n G C}, I_{1}{ }^{*}$ is the complex conjugate of $I_{1} . I_{1}$ is the magnitude of fundamental current.

Under the $d q$-domain IC feedback control, this is equivalent to the L-filter inverter shown in Figure 2 [10], $C_{f}$ and $L_{2}$ are considered as parts of the grid impedance, so the only difference in the signal flow diagram between GC and IC feedback is the green area illustrated in Figure 4. Under the $\alpha \beta$-domain GC feedback control, the only difference of signal flow diagram between $d q$ - and $\alpha \beta$-domain control lies in the blue area depicted in Figure 4. Similarly, their inverter output impedances are modeled as follows:

$$
\begin{gathered}
Z_{p}{ }^{I C}(s)=\frac{K_{m} V_{d c} e^{-s 1.5 T_{s}}\left[H_{i}\left(s-j 2 \pi f_{1}\right)-j K_{d}\right] G_{i}(s)+s L_{1}}{1-K_{m} V_{d c} e^{-s 1.5 T_{s}}\left[H_{i}\left(s-j 2 \pi f_{1}\right)-j K_{d}+M_{n I C}[d c] / I_{1}\right] T_{p}(s) I_{1}} \\
Z_{n}{ }^{I C}(s)=\frac{K_{m} V_{d c} e^{-s 1.5 T_{s}}\left[H_{i}\left(s+j 2 \pi f_{1}\right)+j K_{d}\right] G_{i}(s)+s L_{1}}{1-K_{m} V_{d c} e^{-s 1.5 T_{s}}\left[H_{i}\left(s+j 2 \pi f_{1}\right)+j K_{d}+M_{n I C}[d c] / I_{1}{ }^{*}\right] T_{n}(s) I_{1}{ }^{*}}
\end{gathered}
$$

where:

$$
M_{n I C}[d c]=\left[V_{1}+j w_{1} L_{1} I_{1} e^{j \phi_{i 1}}\right] \cdot\left[K_{m} V_{d c} e^{-s 1.5 T_{s}}\right]^{-1}
$$

and $M_{n I C}{ }^{*}$ is the complex conjugate of $M_{n I C}$.

$$
\begin{gathered}
Z_{p G C}{ }^{\alpha \beta}(s)=\frac{K_{m} V_{d c} e^{-s 1.5 T_{s}} H_{r}(s) G_{i}(s)+P_{1} G C(s)}{P_{2} G C(s)-K_{m} V_{d c} e^{-s 1.5 T_{s}} H_{r}(s)\left(I_{d r} \pm j I_{q r}\right) T_{p}(s)} \\
Z_{n G C}{ }^{\alpha \beta}(s)=\frac{K_{m} V_{d c} e^{-s 1.5 T_{s}} H_{r}(s) G_{i}(s)+P_{1} G C(s)}{P_{2} G C(s)-K_{m} V_{d c} e^{-s 1.5 T_{s}} H_{r}(s)\left(I_{d r} \mp j I_{q r}\right) T_{n}(s)} \\
Z_{p I C}{ }^{\alpha \beta}(s)=\frac{K_{m} V_{d c} e^{-s 1.5 T_{s}} H_{r}(s) G_{i}(s)+s L_{1}}{1-K_{m} V_{d c} e^{-s 1.5 T_{s}} H_{r}(s)\left(I_{d r} \pm j I_{q r}\right) T_{p}(s)} \\
Z_{n I C}{ }^{\alpha \beta}(s)=\frac{K_{m} V_{d c} e^{-s 1.5 T_{s}} H_{r}(s) G_{i}(s)+s L_{1}}{1-K_{m} V_{d c} e^{-s 1.5 T_{s}} H_{r}(s)\left(I_{d r} \mp j I_{q r}\right) T_{n}(s)}
\end{gathered}
$$

\subsection{Impedance Models Verification}

By means of Plecs, we apply a frequency sweep method to verify the modeled impedances. The parameters for the simulation are listed in Table 1.

Figures 7-10 show the frequency response under different control schemes. The red solid line and the blue broken line represent the positive- and negative-sequence models, respectively; the black dashed line and the pink dashed line show the positive- and negative-sequence simulations, respectively. It can be concluded that the modeled impedances are verified well by the simulation, which validates the accurate modeling approach of the output impedances of the grid-connected inverters. It can be seen that differences between positive-sequence and negative-sequence impedance appear at lower frequencies; this is because of the shifting of effects of $\pm j w_{1}$ that makes them different [1]. Additionally, we can see that the main difference between GC and IC control lies at frequencies where $s>>j w_{1}$ as seen comparing Figure 7 with Figure 8 , or Figure 9 with Figure 10. The main difference between $d q$ - and $\alpha \beta$-domain control locates at lower frequencies, as seen by comparison of Figure 7 with Figure 9, or of Figure 8 with Figure 10. 
Table 1. Parameters of the simulation of grid-connected inverters.

\begin{tabular}{ccc}
\hline Parameter & Symbol & Value \\
\hline$d q$-domain Current Control Compensator & $H_{i}(\mathrm{~s})=K_{p}+K_{i} / s$ & $K_{p}=0.04, K_{i}=20$ \\
$\alpha \beta$-domain Current Control Compensator & $H_{r}(\mathrm{~s})=K_{p}+2 K_{i} \mathrm{~s} /\left(s^{2}+w_{1}^{2}\right)$ & $K_{p}=0.04, K_{i}=20$ \\
SRF-PLL Compensator & $H_{P L L}(\mathrm{~s})=\left(K_{p}+K_{i} / s\right) / s$ & $K_{p}=2.98, K_{i}=1990$ \\
Decoupling Gain & $K_{d}$ & 0.0052 \\
Fundamental Grid Voltage & $V_{1}$ & $156 \mathrm{~V}$ \\
Fundamental Frequency & $f_{1}$ & $50 \mathrm{~Hz}$ \\
DC Bus Voltage & $V_{d c}$ & $550 \mathrm{~V}$ \\
PWM Gain & $K_{m}$ & $1 / 2$ \\
Grid Inductance & $L_{g}$ & $5 \mathrm{mH}$ \\
Converter-side inductance & $L_{1}$ & $2 \mathrm{mH}$ \\
Grid-side inductance & $L_{2}$ & $0.5 \mathrm{mH}$ \\
Filter Capacitor & $C_{f}$ & $30 \mathrm{uF}$ \\
Damping feedback coefficient & $K_{i}$ & 33.3 \\
Fundamental current & $I_{1}$ & $10 \mathrm{~A}$ \\
Phase of the Current & $\Phi_{i 1}$ & 0 \\
Voltage Sampling Period & $T_{v}$ & $50 \mathrm{us}$ \\
Current Sampling Period & $T_{i}$ & $25 \mathrm{us}$ \\
Switching Period & $T_{s}$ & $50 \mathrm{us}$ \\
\hline
\end{tabular}

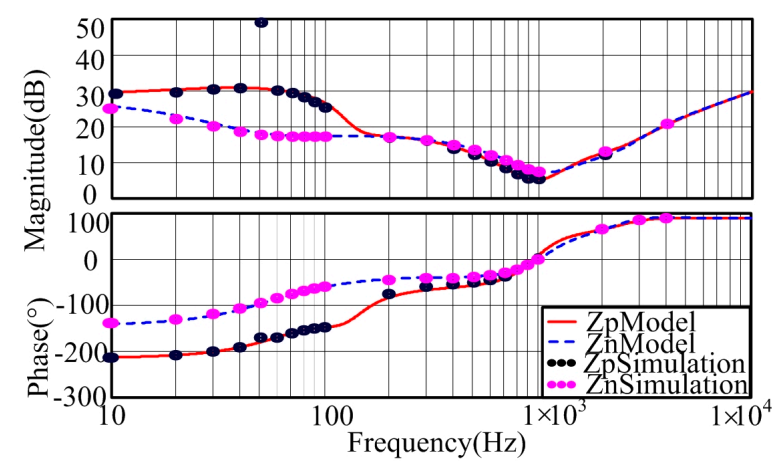

Figure 7. Impedance response with $d q$-domain GC control.

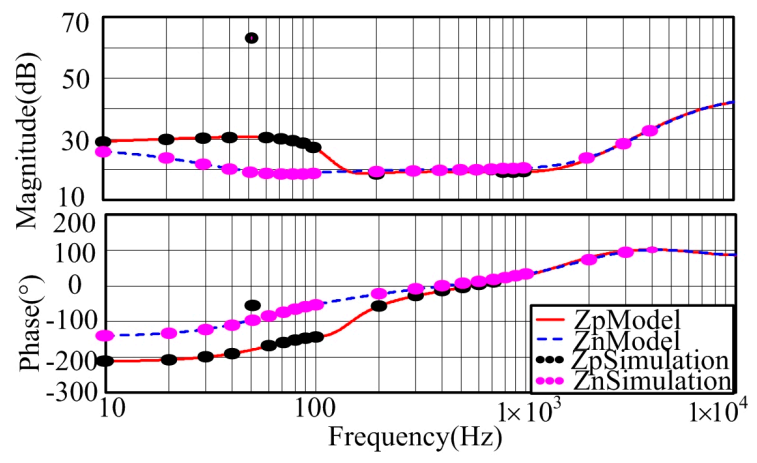

Figure 8. Impedance response with $d q$-domain IC control. 


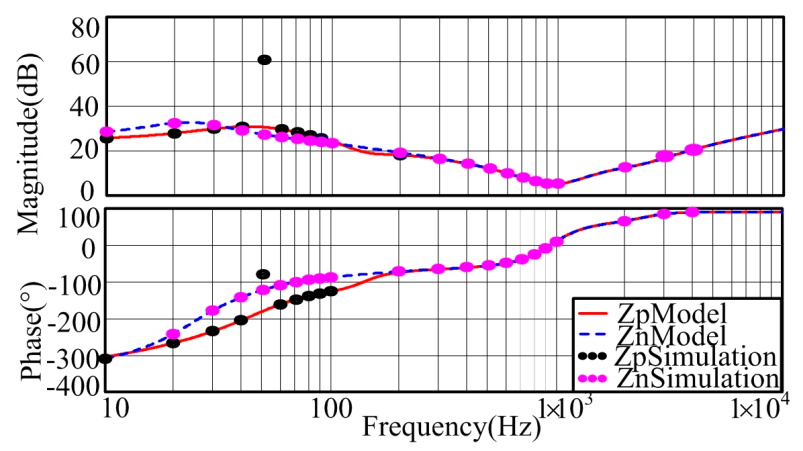

Figure 9. Impedance response with $\alpha \beta$-domain GC control.

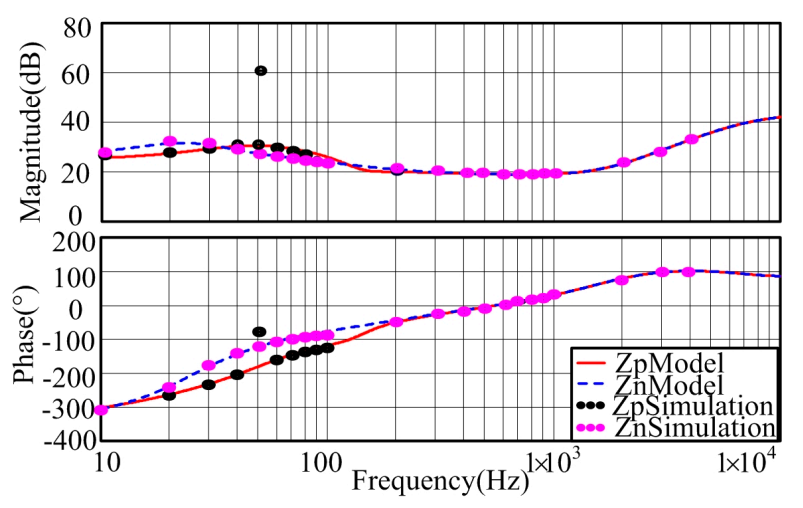

Figure 10. Impedance response with $\alpha \beta$-domain IC control.

\subsection{Impedance Analysis}

Different control schemes present some differences in their corresponding determined frequency ranges. In order to find out the reason why the output impedances show some differences, as depicted in Figures 7-10, here we analyze the dominant factor(s) in different frequency ranges to recognize some existing resonance phenomena better and meanwhile demonstrate indirectly the main difference of different control schemes. Here we take the positive-sequence impedance under the $\alpha \beta$-domain GC feedback control as an example to characterize the impedance in the high, medium and low frequency ranges.

Referring to the high-frequency $(>500 \mathrm{~Hz})$ characteristics of the output impedance, Figure 11 shows the comparison of the whole impedance model and the simplified model as in (22). The simplified model just considers the filter part. It can be concluded that the simplified model can describe the high-frequency impedance characteristics well, which indicates that high-frequency characteristics mainly lie in the filter.

$$
Z_{p}^{\alpha \beta}(s)=\frac{P_{1}(s)}{P_{2}(s)}=\frac{L_{1} L_{2} C_{f} s^{3}+\left(L_{1}+L_{2}\right) s}{L_{1} C_{f} s^{2}+1}
$$




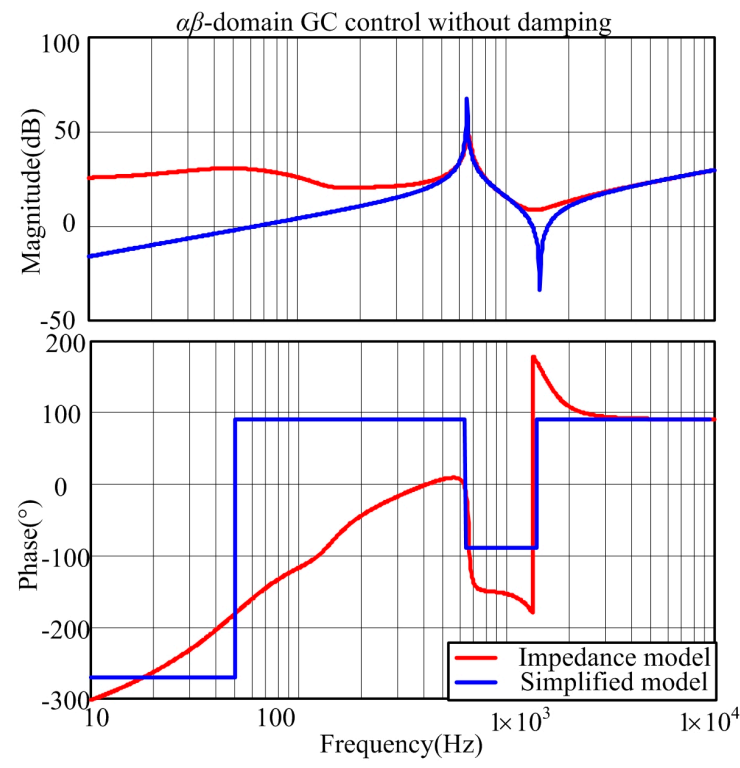

Figure 11. Impedance comparison in high-frequency range.

As for the medium-frequency $(200-500 \mathrm{~Hz})$ characteristics of the output impedance, Figure $12 \mathrm{a}$ shows the comparison of the whole impedance model as in (18) and the simplified model as in (23) derived from neglecting the PLL in (18). Figure 12b depicts the impedance response with different current controller parameters. It can be seen that the simplified model can represent the medium-frequency impedance characteristic well, and the adjustment of current controller parameters has a great influence on the medium-frequency impedance characteristics $[1,6]$, which illustrates that the medium-frequency characteristics are not related with PLL and they are determined mainly by the current controller parameters. Increasing the proportional and integral coefficients can improve the magnitude of the medium-frequency impedance greatly. However it deteriorates the phase in high-frequency range.

$$
Z_{p}^{\alpha \beta}(s)=\frac{K_{m} V_{d c} e^{-s 1.5 T_{s}} H_{r}(s) G_{i}(s)+P_{1} G C(s)}{P_{2} G C(s)}
$$
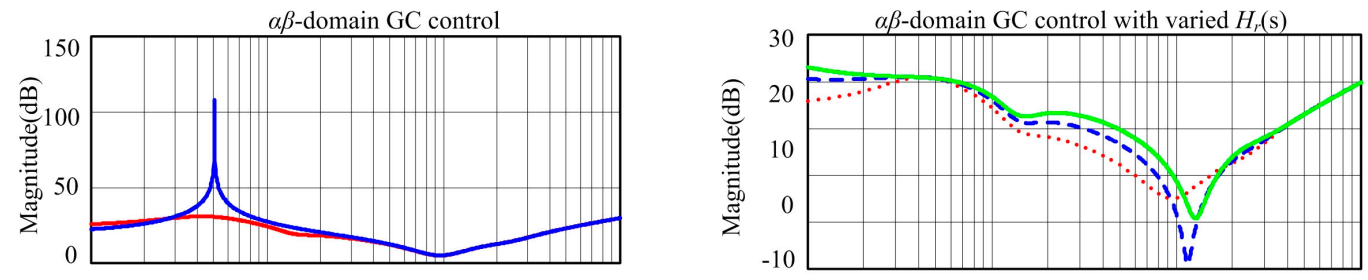

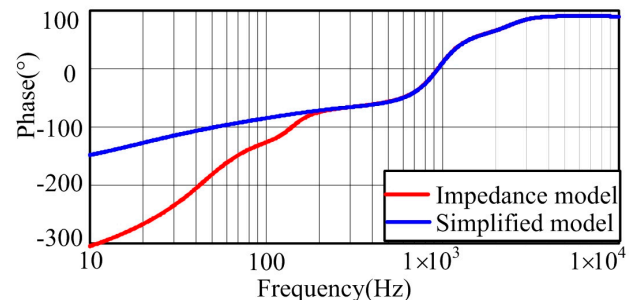

(a)

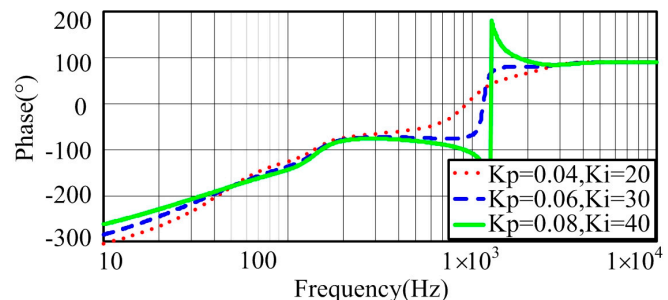

(b)

Figure 12. Impedance comparison in medium-frequency range. (a) Impedance comparison in medium-frequency range; (b) Impedance response with different current controller parameters. 
With regard to the low-frequency $(30-200 \mathrm{~Hz})$ characteristics of the output impedance, Figure 13 depicts the impedance response with different PLL controller parameters. It can be seen that the adjustment of PLL controller parameters has a great influence on the low-frequency impedance characteristics $[1,10]$. Considering the results of Figures $12 \mathrm{~b}$ and 13 , it reveals that PLL contributes a lot to the low-frequency characteristics. Decreasing the bandwidth of the PLL controller can improve the phase of the low-frequency impedance.

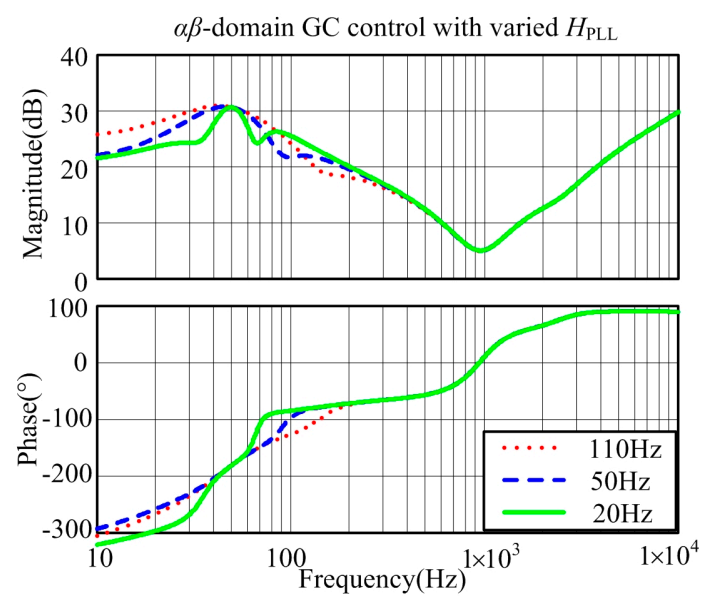

Figure 13. Impedance response with different PLL controller parameters.

From the above analysis and considering the modeling process, since the difference between GC and IC feedback lies in the filter, it can be concluded that the essential difference under different current sampling schemes locates in the high-frequency range; as the main difference between $\alpha \beta$-domain control and $d q$-domain control is the current control loop including the current controller and the PLL, the impedances under different reference frame present different characteristics both in mediumand low-frequency range. The theoretical results are consistent with the model comparison shown in Figures $7-10$.

\section{Stability Analysis}

\subsection{Stability Criterion}

According to the impedance-based stability criterion in [5], the grid-connected inverter is equivalent to the circuit depicted in Figure 14. If we assume that the inverter is stable when connected to a grid with zero impedance, and the grid is stable when an ideal current source with infinite impedance is connected to it, then the interconnected system is stable only if the impedance ratios $Z_{g} / Z_{0}$ conform with the Nyquist stability criterion. Considering a three-phase system, both the positive- and negative-sequence impedance should conform with this criterion, but the positive-sequence impedance turns out to be more critical in terms of stability [21]. Therefore the following analysis mainly aims at comparing the impedance ratio $Z_{g} / Z_{0 p}$. In order to compare the stability under different control schemes, the grid impedance is simplified as varied inductors, which is representative in stability issues associated with variations of grid impedance [11].

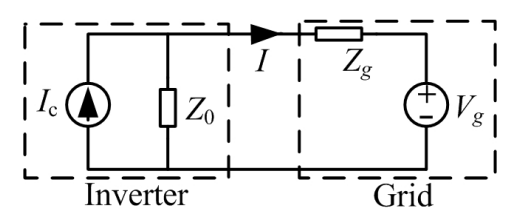

Figure 14. Small-signal representation of inverter-grid system. 


\subsection{Stability Analysis of Inverter-Grid System with Different Current Sampling Schemes}

To the inverter-grid system with different current sampling schemes, according to Figures 1 and 2, their gird impedances are expressed as:

$$
\begin{gathered}
Z_{g}{ }^{G C}(s)=s L_{g} \\
Z_{g}{ }^{I C}(s)=\frac{1}{1 /\left(s L_{2}+s L_{g}\right)+s C_{f}}
\end{gathered}
$$

Combing the grid impedance in Equations (24) and (25) and the output impedance models of the inverter in Equations (9) and (15), Figure 15 shows the impedance-based stability analysis results under different sampling currents when $L_{g}=5 \mathrm{mH}$, where it can be concluded that the impedance ratios $Z_{g} / Z_{0}$ conform with the Nyquist stability criterion in these two cases (the stable margin is sufficient), which indicates the inverter-grid system is stable when $L_{g}=5 \mathrm{mH}$. Figure 16 depicts the grid-connected simulation currents; there is no resonance in the currents, which validates the accuracy of the impedance-based stability analysis results in Figure 15.

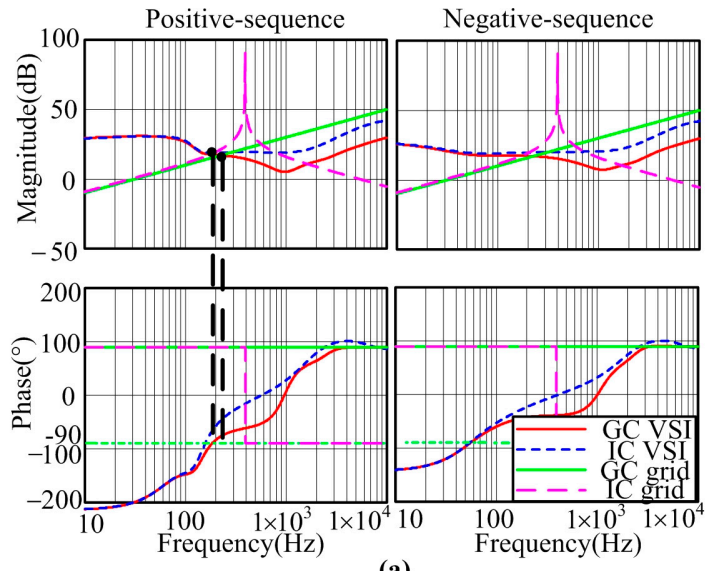

(a)

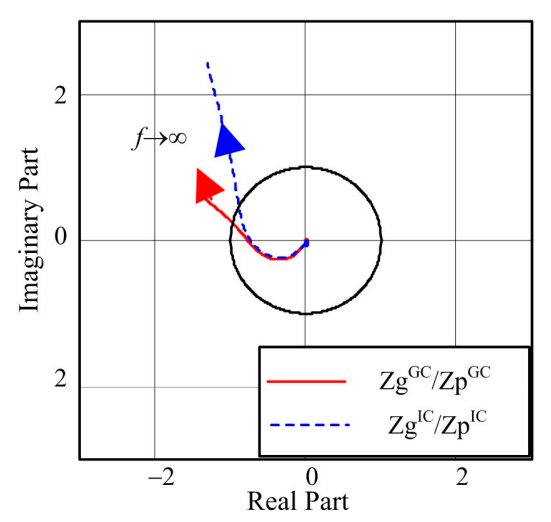

(b)

Figure 15. Impedance-based stability analysis results under different sampling currents when $L_{\mathrm{g}}=5 \mathrm{mH}$. (a) Impedances of VSI and the grid; (b) Nyquist plot of impedance ratio.

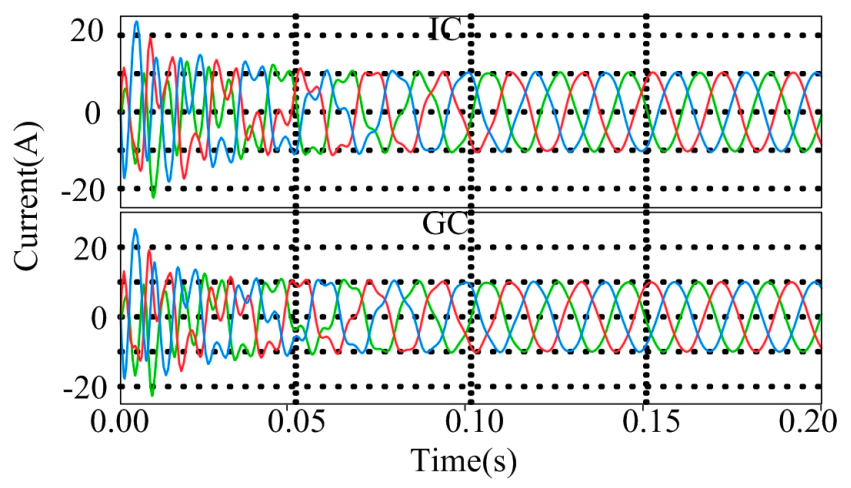

Figure 16. Grid-connected currents under different sampling currents when $L_{g}=5 \mathrm{mH}$.

In order to compare the resonance phenomena under different sampling currents clearly, here we list the impedance-based stability analysis results in Table 2. It can be derived that the inverter-grid system begins resonating when the grid inductor increases to $6 \mathrm{mH}$ under these two current sampling schemes. It can also be inferred that different sampling schemes matter little to the resonance resulting from the varied grid impedance, because different sampling schemes only vary in the high frequency, 
which can be obtained from the impedance analysis in Section 2.3, so for the lower-frequency stability resulting from weak grid conditions, their difference is too small to be ignored. The main difference is that GC control can ensure the power factor, while the IC control doesn't need damping. Here we think more about the power factor, so GC control is better.

Table 2. Stability referring to different current sampling schemes.

\begin{tabular}{cccccc}
\hline $\begin{array}{c}\text { Grid Inductor } \\
(\mathbf{m H})\end{array}$ & $\begin{array}{c}\text { Sampling } \\
\text { Schemes }\end{array}$ & $\begin{array}{c}\text { Crossover } \\
\text { Frequency (Hz) }\end{array}$ & $\begin{array}{c}\text { Phase } \\
\left.\text { Margin } \mathbf{(}^{\circ}\right)\end{array}$ & $\begin{array}{c}\text { Magnitude } \\
\text { Margin (dB) }\end{array}$ & Stability Mode \\
\hline 5 & GC & 226 & 14 & 2.25 & stable \\
5 & IC & 190 & 28 & 2.70 & stable \\
6 & GC & 195 & 5 & 0.7 & unstable \\
6 & IC & 166 & 9 & 0.9 & unstable \\
\hline
\end{tabular}

\subsection{Stability Analysis of Inverter-Grid System with Different Current Reference Frame}

Here the stability analysis is implemented under GC sampling. For the inverter-grid system with different current reference frame, the gird impedances are both expressed as in (24). We take the same analysis method as described in Section 3.2. Combing the grid impedance in Equation (24) and the output impedance models in Equations (9) and (18), Table 3 shows the impedance-based stability analysis results. It can be seen that the resonance phenomena under different reference frame varies greatly. When the grid inductor increases to $6 \mathrm{mH}$, the $d q$-domain feedback control displays resonance and the $\alpha \beta$-domain feedback control is still stable, until the grid inductor increases to $8 \mathrm{mH}$, where the $\alpha \beta$-domain feedback control shows resonance, which illustrates that the $\alpha \beta$-domain feedback control has better performance compared to the $d q$-domain feedback control when the grid impedance varies. This can be explained by the impedance analysis in Section 2.3-the impedance under different reference frame varies in lower frequency, so their robustness is apparent under the weak grid conditions.

Table 3. Stability referring to different current reference frame.

\begin{tabular}{cccccc}
\hline $\begin{array}{c}\text { Grid Inductor } \\
(\mathbf{m H})\end{array}$ & $\begin{array}{c}\text { Reference } \\
\text { Frame }\end{array}$ & $\begin{array}{c}\text { Crossover } \\
\text { Frequency } \mathbf{( H z )}\end{array}$ & $\begin{array}{c}\text { Phase } \\
\text { Margin }\left(^{\circ}\right)\end{array}$ & $\begin{array}{c}\text { Magnitude } \\
\text { Margin (dB) }\end{array}$ & Stability Mode \\
\hline 5 & $d q$ & 226 & 14 & 2.25 & stable \\
5 & $\alpha \beta$ & 239 & 20 & 5.08 & stable \\
6 & $d q$ & 195 & 5 & 0.7 & unstable \\
6 & $\alpha \beta$ & 211 & 17 & 3.5 & stable \\
7 & $d q$ & 172 & -6 & -0.7 & unstable \\
7 & $\alpha \beta$ & 187 & 13 & 2.2 & stable \\
8 & $\alpha \beta$ & 168 & 7 & 1.0 & unstable \\
\hline
\end{tabular}

\subsection{Stability Analysis of Inverter-Grid System with Different Control Parameters}

From the above analysis, we conclude that the $\alpha \beta$-domain GC feedback control is verified to be the best control scheme for the VSI with LCL-filter connected to a weak grid. To improve the robustness, here we analyze the contribution of regulating the control parameters to adjust the grid impedance variation. Referring to the control loop of the inverter-grid system, it mainly consists of the current controller and the PLL controller. From the analysis in Section 2.3, it can be drawn that increasing the controller bandwidth can enlarge the magnitude of the inverter impedance greatly in the medium-frequency range, while decreasing the PLL bandwidth can enlarge the phase of the inverter impedance remarkably in the low-frequency range. Considering the two control parameter regulating methods, here we compare their effects to find the more efficient way to eliminate resonance. 
Tables 4 and 5 show the resonance analysis results by means of the impedance-based stability analysis approach. It can be seen that by increasing the current controller bandwidth by $40 \%$ or decreasing the PLL controller bandwidth by $30 \%$ when the grid inductor is $8 \mathrm{mH}$, the resonant currents in Table 3 become stable, as shown in the tables, but in the first case, the system becomes resonant when the grid inductor grows to $10 \mathrm{mH}$, and in the second case, the resonance emerges until the grid inductor increases to $11 \mathrm{mH}$. Therefore it is more efficient to decrease the PLL bandwidth to improve the stability. The disadvantage is that if the PLL bandwidth is lower, its dynamic response will be slower, but the increase of the current controller bandwidth may result in higher-frequency resonances.

Table 4. Stability state when increasing the current controller bandwidth by $40 \%$.

\begin{tabular}{ccccc}
\hline $\begin{array}{c}\text { Grid Inductor } \\
(\mathbf{m H})\end{array}$ & $\begin{array}{c}\text { Crossover Frequency } \\
(\mathbf{H z})\end{array}$ & $\begin{array}{c}\text { Phase Margin } \\
\left({ }^{\circ}\right)\end{array}$ & $\begin{array}{c}\text { Magnitude Margin } \\
(\mathbf{d B})\end{array}$ & Stability Mode \\
\hline 8 & 228 & 15 & 12 & stable \\
9 & 205 & 12 & 11 & stable \\
10 & 184 & 8 & 10 & unstable \\
\hline
\end{tabular}

Table 5. Stability state when decreasing the PLL controller bandwidth by $30 \%$.

\begin{tabular}{ccccc}
\hline $\begin{array}{c}\text { Grid Inductor } \\
(\mathbf{m H})\end{array}$ & $\begin{array}{c}\text { Crossover Frequency } \\
(\mathbf{H z})\end{array}$ & $\begin{array}{c}\text { Phase Margin } \\
\left({ }^{\circ}\right)\end{array}$ & $\begin{array}{c}\text { Magnitude Margin } \\
(\mathbf{d B})\end{array}$ & Stability Mode \\
\hline 8 & 185 & 15 & 12 & stable \\
9 & 171 & 14 & 15 & stable \\
10 & 158 & 11 & 14 & stable \\
11 & 145 & 8 & 13 & unstable \\
\hline
\end{tabular}

\section{Experimental Results}

To confirm the theoretical analysis in Section 3, a series of experiments were implemented on a $3 \mathrm{~kW}$ wind power experimental platform depicted in Figure 17. On this platform, the grid inductor has values of $2,4,6,8$ and $10 \mathrm{mH}$. By choosing different values, we can simulate the variation of the grid impedance to compare the effects of different control schemes under weak grid conditions.

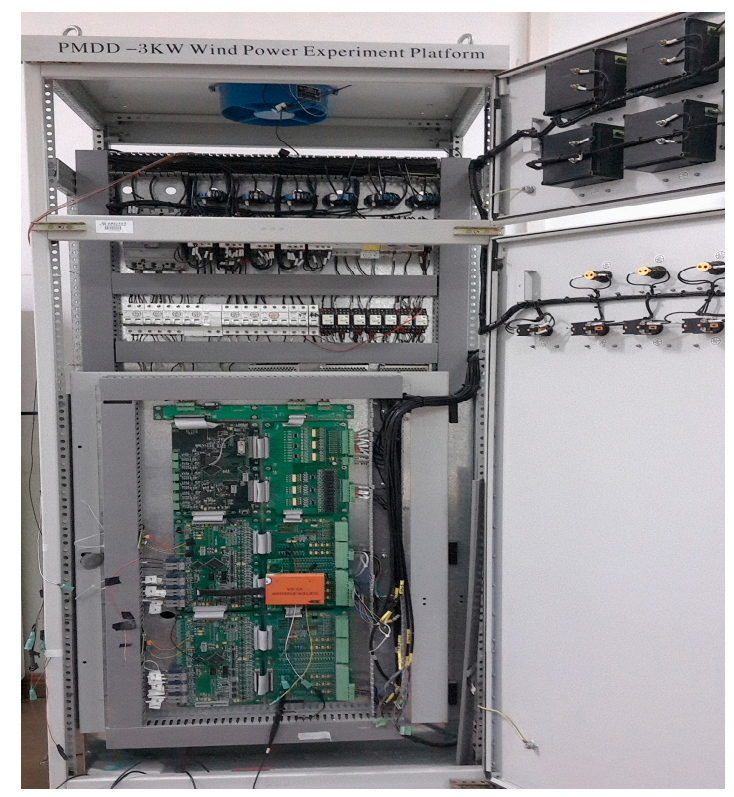

Figure 17. The experimental platform of stability analysis in inverter-grid system. 
Figure 18 shows the stability comparison under different current sampling schemes, where it can be seen that when $L_{g}=4 \mathrm{mH}$, the current waveform is sinusoidal under both the IC and GC sampling schemes, and when the grid inductor increases to $6 \mathrm{mH}$, the currents are resonant together, which indicates that different sampling schemes contribute little to the resonance resulting from varied grid impedance. Although the THD under IC sampling is smaller than in the GC sampling scheme, the difference is so small that it can be neglected.

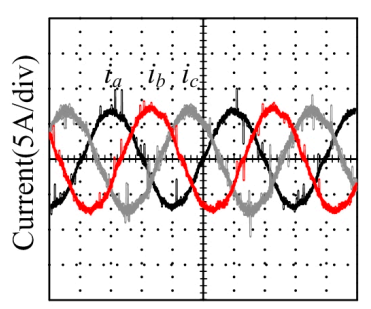

Time $(5 \mathrm{~ms} / \mathrm{div})$

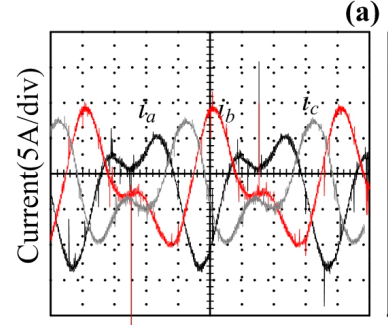

Time(5ms/div)

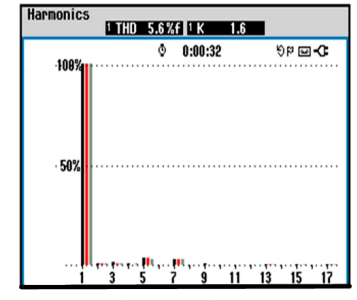

Harmonic order

a)

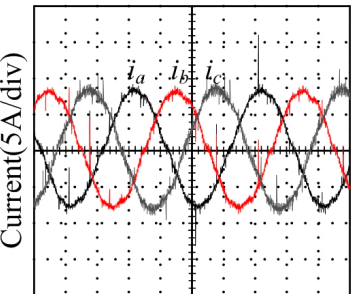

Time $(5 \mathrm{~ms} / \mathrm{div})$

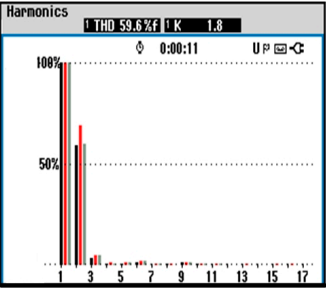

Harmonic order

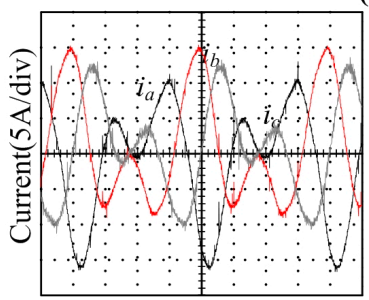

Time(5ms/div)

(c)

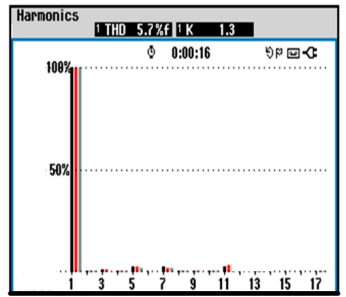

(b)

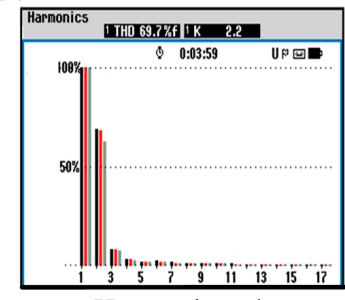

Harmonic order

(d)

Figure 18. Grid-connected currents comparison under different current sampling schemes with varied grid impedance. (a) Grid-connected currents under IC sampling with $L_{g}=4 \mathrm{mH}$; (b) Grid-connected currents under GC sampling with $L_{g}=4 \mathrm{mH}$; (c) Grid-connected currents under IC sampling with $L_{g}=6 \mathrm{mH}$; (d) Grid-connected currents under GC sampling with $L_{g}=6 \mathrm{mH}$.

Figure 19 depicts the stability comparison with GC sampling under different current reference frames, where we can draw the conclusions that when $L_{g}=2 \mathrm{mH}$, the current waveform is stable both under the $d q$-domain and $\alpha \beta$-domain control schemes, that when the grid inductor increases to $4 \mathrm{mH}$, the currents display apparent resonance under $d q$-domain control, and the currents under $\alpha \beta$-domain control become unstable when $L_{g}=6 \mathrm{mH}$, which indicates that the $\alpha \beta$-domain control is more adaptable to the weak grid than $d q$-domain control.

Aiming at eliminating the resonance seen in Figure 19e, Figure 20 compares the effects of regulating the current controller and PLL controller. It can be concluded that decreasing the PLL controller bandwidth by $41 \%$ can eliminate the resonance absolutely and increasing the current controller bandwidth by $44 \%$ can only improve the resonance phenomena, so decreasing the PLL controller bandwidth is more effective to improve the stability in inverter-grid systems. 


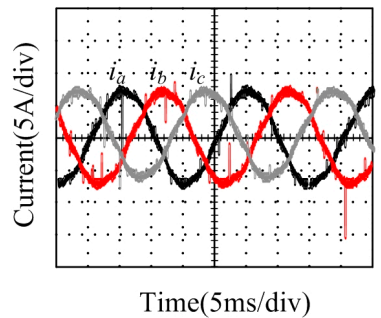

(a)

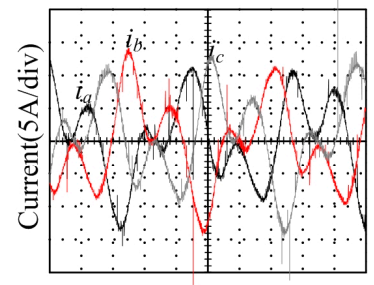

Time(5ms/div)

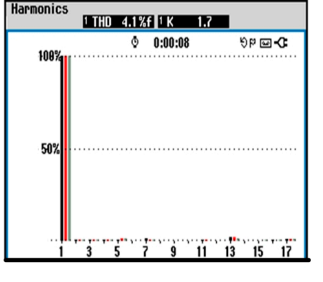

Harmonic order

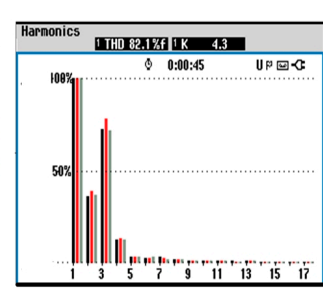

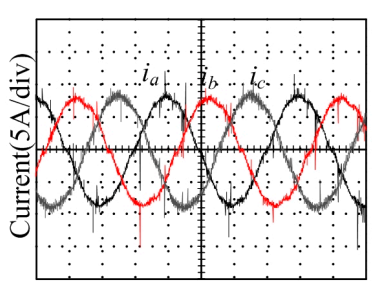

Time(5ms/div)

(b)

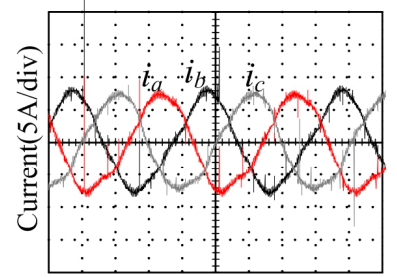

Time $(5 \mathrm{~ms} / \mathrm{div})$

(d)

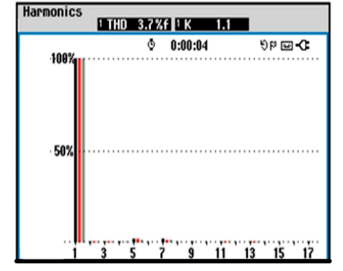

Harmonic order

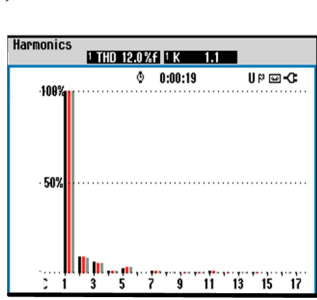

Harmonic order (c)

Harmonic order

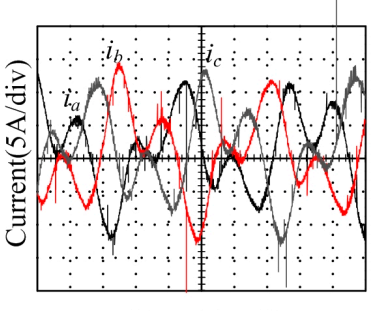

Time( $5 \mathrm{~ms} / \mathrm{div})$

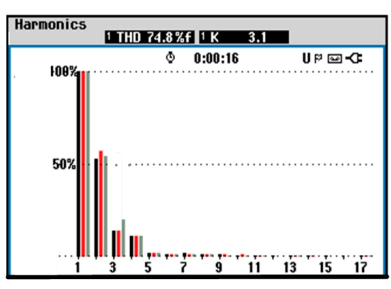

Harmonic order

(e)

Figure 19. Grid-connected currents comparison under different current reference frame with varied grid impedance. (a) Grid-connected currents under $d q$-domain control with $L_{g}=2 \mathrm{mH}$; (b) Grid-connected currents under $\alpha \beta$-domain control with $L_{g}=2 \mathrm{mH}$; (c) Grid-connected currents under $d q$-domain control with $L_{g}=4 \mathrm{mH}$; (d) Grid-connected currents under $\alpha \beta$-domain control with $L_{g}=4 \mathrm{mH}$; (e) Grid-connected currents under $\alpha \beta$-domain control with $L_{g}=6 \mathrm{mH}$.

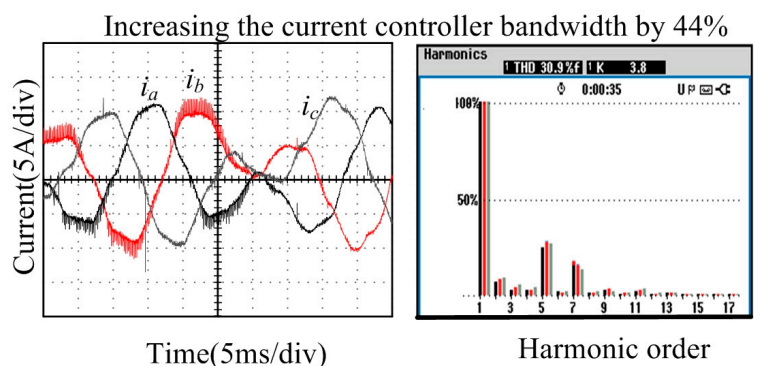

(a)

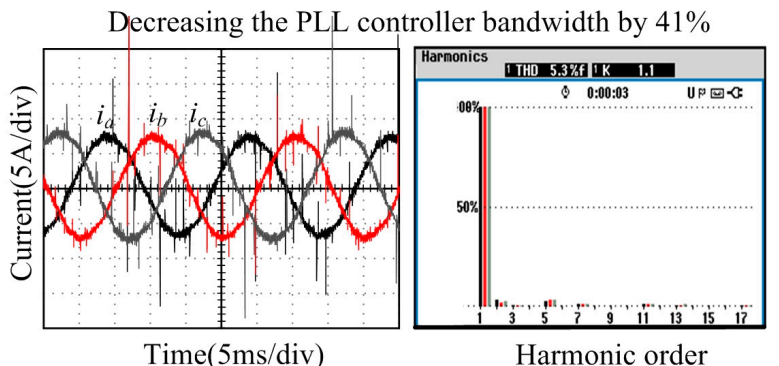

(b)

Figure 20. Grid-connected currents comparison under different control parameters with varied grid impedance. (a) Grid-connected currents under current controller regulating with $L_{g}=6 \mathrm{mH}$; (b) Grid-connected currents under PLL controller regulating with $L_{g}=6 \mathrm{mH}$.

\section{Conclusions}

For the three-phase grid-connected inverter with LCL filter, according to different sampling positions and different reference frames, there mainly exist four control schemes. In order to compare their robustness towards the variation of the grid impedance, this paper models the output impedances of the inverter with different control schemes directly in the frequency domain using the harmonic linearization method. Impedance analysis reveals that their high-frequency characteristics mainly lie in the filter, the medium-frequency characteristics are influenced by the current controller parameters, 
and PLL contributes a lot to the low-frequency characteristics. The impedances built in this paper can perfectly demonstrate the differences among different control schemes. Out of consideration of adapting to a weak grid, by means of the impedance-based stability analysis, the $\alpha \beta$-domain GC feedback control is verified to have the strongest robustness and regulating the PLL control parameters can effectively improve the stability. Although the effect of PLL controller regulation is limited, the simplified impedance models and derived conclusion can make a great contribution to develop adaptive controls to cope with the variation of grid impedance and improve the stability of paralleled grid-connected VSIs from the internal mechanism.

Acknowledgments: This work was from the project Research on Integrated Evaluation of the Impact of Connected Distributed Generation on Heilongjiang Distributed Networks, supported by State Grid.

Author Contributions: Yanxue Yu was responsible for the theoretical derivation, simulation and paper writing. Haoyu Li proposed the main idea and analysis method. Zhenwei Li and Zhou Zhao designed the experiments.

Conflicts of Interest: The authors declare no conflict of interest.

\section{References}

1. Cespedes, M.; Sun, J. Impedance Modeling and Analysis of Grid-Connected Voltage-Source Converters. IEEE Trans. Power Electron. 2014, 29, 1254-1261. [CrossRef]

2. Roinila, T.; Vilkko, M.; Sun, J. Online Grid Impedance Measurement Using Discrete-Interval Binary Sequence Injection. IEEE J. Emerg. Sel. Top. Power Electron. 2013, 2, 1-8.

3. Cespedes, M.; Qi, T.; Sun, J. Development of a grid simulator. In Proceedings of the 2012 IEEE 13th Workshop on Control and Modeling for Power Electronics (COMPEL), Kyoto, Japan, 10-13 June 2012; pp. 1-8.

4. Chen, X.; Sun, J. Characterization of inverter-grid interactions using a hardware-in-the-loop system test-bed. In Proceedings of the 2011 IEEE 8th International Conference on Power Electronics and ECCE Asia (ICPE \& ECCE), Jeju, Korea, 30 May-3 June 2011; pp. 2180-2187.

5. Sun, J. Impedance-Based Stability Criterion for Grid-Connected Inverters. IEEE Trans. Power Electron. 2011, 26, 3075-3078. [CrossRef]

6. Liserre, M.; Teodorescu, R.; Blaabjerg, F. Stability of photovoltaic and wind turbine grid-connected inverters for a large set of grid impedance values. IEEE Trans. Power Electron. 2006, 21, 263-272. [CrossRef]

7. Hoseinzadeh, B.; Bak, C.L. Admittance modeling of voltage and current controlled inverter for harmonic instability studies. In Proceedings of the 2016 IEEE Power and Energy Society General Meeting (PESGM), Boston, MA, USA, 17-21 July 2016; pp. 1-5.

8. Yoon, C.; Bai, H.; Beres, R.N.; Wang, X.; Bak, C.L.; Blaabjerg, F. Harmonic Stability Assessment for Multiparalleled, Grid-Connected Inverters. IEEE Trans. Sustain. Energy 2016, 7, 1388-1397. [CrossRef]

9. Jiao, J.; Nelms, R.M. Regulating output impedance using a PI controller to improve the stability of a single phase inverter under weak grid. In Proceedings of the 2016 IEEE 16th International Conference on Environment and Electrical Engineering (EEEIC), Florence, Italy, 7-10 June 2016.

10. Yu, Y.; Li, H.; Li, Z. Impedance modeling and stability analysis of LCL-type grid-connected inverters with different current sampling schemes. In Proceedings of the 2016 IEEE 8th International Power Electronics and Motion Control Conference, Hefei, China, 22-26 May 2016; pp. 974-981.

11. Li, X.; Wu, X.; Geng, Y. Stability Analysis of Three-phase PV Inverter under Inductive Grid Impedance Condition. Proc. CSEE 2014, 34, 2906-2916.

12. Pan, D.; Ruan, X.; Wang, X.; Yu, H.; Xing, Z. Analysis and Design of Current Control Schemes for LCL-Type Grid-Connected Inverter Based on a General Mathematical Model. IEEE Trans. Power Electron. 2016, 99, 1. [CrossRef]

13. Chen, X.; Gong, C.Y.; Wang, H.Z. Stability analysis of LCL-type grid-connected inverter in weak grid systems. In Proceedings of the 2012 International Conference on Renewable Energy Research and Applications (ICRERA), Nagasaki, Japan, 11-14 November 2012; pp. 1-6.

14. Dannehl, J.; Liserre, M.; Fuchs, F.W. Filter-Based Active Damping of Voltage Source Converters with LCL Filter. IEEE Trans. Ind. Electron. 2011, 58, 3623-3633. [CrossRef]

15. Wang, X.; Blaabjerg, F.; Loh, P.C. Grid-Current-Feedback Active Damping for LCL Resonance in Grid-Connected Voltage-Source Converters. IEEE Trans. Power Electron. 2016, 31, 213-223. [CrossRef] 
16. Yang, D.; Ruan, X.; Wu, H. Impedance Shaping of the Grid-Connected Inverter with LCL Filter to Improve Its Adaptability to the Weak Grid Condition. IEEE Trans. Power Electron. 2014, 29, 5795-5805. [CrossRef]

17. Chen, X.; Zhang, Y.; Wang, S.; Chen, J.; Gong, C. Impedance-Phased Dynamic Control Method for Grid-Connected Inverters in a Weak Grid. IEEE Trans. Power Electron. 2017, 32, 274-283. [CrossRef]

18. Midtsund, T.; Suul, J.A.; Undeland, T. Evaluation of current controller performance and stability for voltage source converters connected to a weak grid. In Proceedings of the 2010 2nd IEEE International Symposium on Power Electronics for Distributed Generation Systems (PEDG), Hefei, China, 16-18 June 2010; pp. 382-388.

19. Tamrakar, U.; Tonkoski, R.; Ni, Z.; Hansen, T.M.; Tamrakar, I. Current control techniques for applications in virtual synchronous machines. In Proceedings of the 2016 IEEE 6th International Conference on Power Systems (ICPS), New Delhi, India, 4-6 March 2016; pp. 1-6.

20. Althobaiti, M.; Armstrong, M.; Elgendy, A.; Mulolani, F. Three-phase grid connected PV inverters using the proportional resonance controller. In Proceedings of the 2016 IEEE 16th International Conference on Environment and Electrical Engineering (EEEIC), Florence, Italy, 6-8 June 2016; pp. 1-6.

21. Céspedes, M. Modeling and mitigation of harmonic resonance between wind turbines and the grid. In Proceedings of the 2011 IEEE Energy Conversion Congress and Exposition (ECCE), Phoenix, AZ, USA, 17-22 September 2011; pp. 2109-2116.

(C) 2017 by the authors; licensee MDPI, Basel, Switzerland. This article is an open access article distributed under the terms and conditions of the Creative Commons Attribution (CC-BY) license (http://creativecommons.org/licenses/by/4.0/). 